\title{
Fourier Transform Imaging of Spin Vortex Eigenmodes
}

\author{
M. Buess, ${ }^{1,2}$ R. Höllinger, ${ }^{1}$ T. Haug, ${ }^{1}$ K. Perzlmaier, ${ }^{1}$ U. Krey, ${ }^{1}$ D. Pescia, ${ }^{1}$ M. R. Scheinfein, ${ }^{3}$ D. Weiss, ${ }^{1}$ and C. H. Back ${ }^{1}$ \\ ${ }^{1}$ Institut für Experimentelle und Angewandte Physik, Universität Regensburg, Universitätsstrasse 31, 93040 Regensburg, Germany \\ ${ }^{2}$ Laboratorium für Festkörperphysik, Eidgenössische Technische Hochschule Zürich, CH-8093 Zürich, Switzerland \\ ${ }^{3}$ Simon Fraser University, 8888 University Drive, Burnaby BC U5A 156, Canada \\ (Received 25 February 2004; published 12 August 2004; publisher error corrected 13 September 2004)
}

Thin-circular lithographically defined magnetic elements with a spin vortex configuration are excited with a short perpendicular magnetic field pulse. We report the first images of excited magnetic eigenmodes up to third order, obtained by means of a phase sensitive Fourier transform imaging technique. Both axially symmetric and symmetry breaking azimuthal eigenmodes are observed. We observe strong oscillations of the magnetization in the central part of the magnetic elements. The experimental data are in good agreement with micromagnetic simulations.

DOI: 10.1103/PhysRevLett.93.077207

PACS numbers: 75.40.Gb, 75.75.+a, 75.60.-d

Small lithographically defined structures with lateral dimensions on the order of a micron or less can behave like resonators for magnetostatic or spin wave modes excited by magnetic field pulses. Eigenmodes, defined by the boundary conditions imposed by the shape of the structure, are selected from the continuous excitation spectrum[1-3]. These excitations exhibit added complexity when the ground state magnetization configuration is the flux-closed magnetic vortex whose inner core supports perpendicular magnetization components on a nanometer length scale. Singular spin configurations and their dynamics offer an intriguing new pathway toward elementary spin logic operations, related to the possibility of switching the core magnetization $[4,7,8]$.

Here, the multimode excitation spectrum has been measured in the time domain using time resolved scanning Kerr microscopy with $300 \mathrm{~nm}$ spatial resolution. One of the difficulties in identifying the dynamical eigenmodes is that the excitation by a short magnetic field pulse results in a complicated spin motion where several modes are superposed in an intricate way. We have circumvented this difficulty by using a phase sensitive Fourier transformation (FT) technique with appropriate window functions. Frequency domain analysis of the time sequenced images reveals resonances corresponding to the eigenmodes defined by the lithographed elements. As the time domain signal is Fouriertransformed retaining both the amplitude and the phase of the FT, back transformation of the complex FT at resonance allows mode-by-mode reconstruction. Using this back-FT technique, we have identified several axially symmetric modes and nonaxially symmetric azimuthal modes. The presence of eigenmodes breaking the axial symmetry of the ground state is thought to be essential for core switching [8]. In fact, we have observed that the central region of the vortex is changing its sign with time, suggesting that core switching might have been realized.

The samples are single $15 \mathrm{~nm}$ thick ferromagnetic permalloy disks with diameters of 3,4 , and $6 \mu \mathrm{m}$, and produced by $e$-beam evaporation according to a now well established procedure[5]. They were patterned by $e$-beam lithography onto a Si substrate covered by $100 \mathrm{~nm}$ thick $\mathrm{Si}_{3} \mathrm{~N}_{4}$. The disks were capped by $2 \mathrm{~nm}$ Al for corrosion protection since the experiments were performed at ambient pressure. X-ray fluorescence was used to determine the thickness and composition on a wafer. A $500 \mathrm{~nm}$ thick $\mathrm{Cu}$ microcoil was prepared around the element using $e$ beam lithography and electroplating. It surrounds the sample with an inner diameter of $8 \mu \mathrm{m}$ and an outer diameter of $12 \mu \mathrm{m}$. The magnetic ground state of the elements exhibits a flux closure, vortex-like spin configuration[5]. A current pulse of about 100 ps rise time launched into the microcoil provides the perpendicularly oriented magnetic tipping field pulse (with strength $\approx$ 50 Oe) exciting the spin precessional motion $[1,9,10]$. To suppress changes of the reflectivity which are not caused by the magnetization, the excitation is gated with a frequency of about $2 \mathrm{kHz}$ and measured with a lock-in technique. Thus we measure the difference of the polar Kerr signal with and without field pulse. The detected signal is proportional to the change of the magnetization due to the excitation: $\mathrm{I}_{\text {Kerr }} \propto M_{z}(t)-M_{z}(0)$ [11]. Figure 1 shows a stroboscopic sequence (also available as an animated online movie [12]) of polar Kerr microscopy images obtained immediately after application of the field pulse (disk diameter: $6 \mu \mathrm{m}$ ). The time interval between images is $30 \mathrm{ps}$. The image contrast is produced by the $z$ component of the magnetization vector undergoing a spatially non-uniform motion. Notice that our experiment measures changes of $M_{z}$ with respect to the ground state configuration, so that the image of the initial state (the state at time "zero") shows no contrast. Despite the complexity of the sequence, one recognizes an overall periodicity of the motion with maxima (bright) and minima (dark) of $M_{z}$ recurring after a characteristic time of the order of $350 \mathrm{ps}$. The modal structure of the motion is not immediately apparent from Fig. 1 but will emerge from the FT analysis. Notice that in this regime of small 


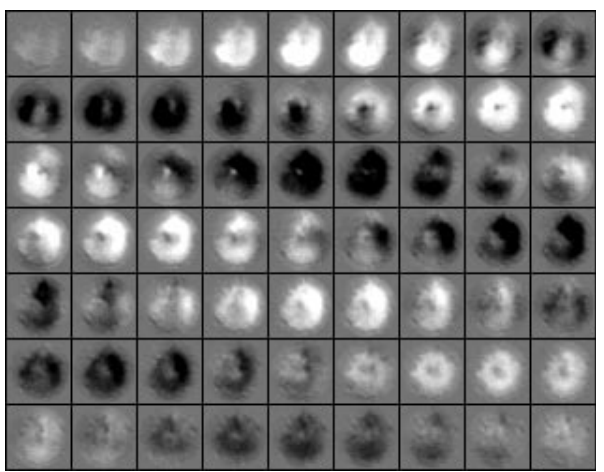

FIG. 1. Temporal evolution of the precessional motion. $M_{z}(t)-M_{z}(t=0)$ is imaged as a function of the time elapsed after the magnetic field pulse. Notice that some images show a central spot which is clearly distinguishable from the surroundings. This feature will be discussed in connection with Fig. 5.

deviations from the ground state the in-plane tangential component of the magnetization vector is, to first order, unaffected by the motion[9,10]. The radial component $M_{r}$ (not measured in the present experiment) is connected to $M_{z}(t)$ by the relation $\dot{M}_{r} \propto M_{z}[10]$, so that the magnetization vector performs an elliptical precession. Thus, in this linear limit, it is sufficient to consider $M_{z}$ in order to have the full knowledge of the modal structure.

A detailed view of the various eigenmodes driving the spin motion in Fig. 1 is obtained by Fourier transforming the time domain signal recorded at each location into the frequency domain. The FT data are reassembled to show spatially resolved maps not only of the amplitude - as in Ref.[2,3] — but also of the phase. The Fourier spectrum consists of a sequence of resonances along the frequency axis corresponding to the eigenmodes. Although the spacing in the frequency domain of the raw data is $1 /\left(T_{\text {end }}-T_{\text {start }}\right)=1 / 2.9 \mathrm{~ns}=0.34 \mathrm{GHz}$, the exact location of the maximum of these resonances can be determined with a higher accuracy of $0.1 \mathrm{GHz}$ when using zerofilling. The typical width of the resonance curves is $1 \mathrm{GHz}$. The values of the resonance frequencies (given in the captions to the figures) have been accurately reproduced by an analytical calculation based on Ref.[10] and by a micromagnetic simulation based on the code developed by one of us[13]. Details of these calculations, which go beyond the scope of this Letter, will be published in a separate Letter[14]. Here images of the amplitude and the phase at resonance of various eigenmodes are displayed in Figs. 2 and 3. We distinguish two types of eigenmodes[15]: those with an axially symmetric spectral weight and phase (Fig. 2) and those breaking the axial symmetry of the ground state (Fig. 3). Only axially symmetric modes are expected if the tipping pulse is uniform over the disk and all geometries are perfectly axially symmetric. Symmetry breaking modes, instead, require, e.g., a non-uniform tipping pulse or a deviation of the sample from a perfect cylindrical shape. Our compu- tation of the field configuration arising from the single turn coil reveals that the tipping pulse has a sizable gradient in the plane of the vortex, owing to the coil opening towards the leads. The maximum difference in tipping field amplitude reaches $30 \%$. As revealed by our micromagnetic simulation[13], this asymmetry is capable of exciting non-axially symmetric modes. Fourieramplitude (left) and -phase (right) images of the three low-lying axially symmetric modes are displayed in Fig. 2(a)-2(c). The left hand side of each image shows the result of the micromagnetic calculation, the right hand side is the amplitude (or phase) of the Fourier transform of the experimental time domain images. The modes can be classified according to the number of nodes: the fundamental and highest-amplitude mode has a node only in the center of the disk and at its border 2(a). The phase is uniform over the disk. The next mode has a node at approximately half distance between the core and the boundary 2(b). Across the node the phase changes by $\pi$, just as in any standing wave. Finally, we observe a third mode with two nodes within the disk 2(c) and with $\pi$ phase jump across each node. This modal structure is well described by the first order Bessel function $J_{1}\left(k_{i} r\right)$, where

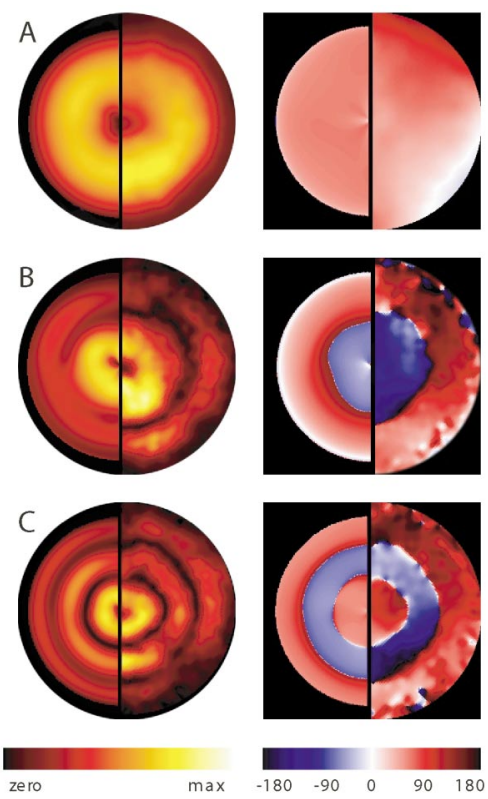

FIG. 2 (color). Fourier transform of the axially symmetric eigenmodes. FT of Fig. 1 at each location results in a Fourier spectrum with five resonances (width: $\approx 1 \mathrm{GHz}$ ). In (a)-(c): Fourier amplitude (left) and Fourier phase (right) at resonance for modes with radial symmetry. Their frequencies are $2.8 \mathrm{GHz}$ (a), $3.9 \mathrm{GHz}$ (b) and $4.5 \mathrm{GHz}$ (c). (a) has the largest spectral weight, i.e., it is dominating the motion and is responsible for the overall periodicity apparent from Fig. 1. The left hand side of the images reports the results of our micromagnetic simulation; the right hand side are the experimental data. Because of our finite spatial resolution the experimental images extend over the border of the disk. 
$k_{i}$ assumes discrete values determined by the boundary conditions[10] and $r$ is the radius. Such modes are natural basis functions in a circular membrane[15].

Figure 3(a) shows Fourier amplitude (left) and Fourier phase (right) at resonance of the lowest lying non-axially symmetric mode. This mode has two mutually orthogonal radial nodal lines (left). In going across each node the phase changes by $\pi$ (right). Images of this mode in the time domain can be reconstructed by back Fourier transforming the amplitude and phase maps of Fig. 3(a). Some snapshots of the time sequence are shown in Fig. 3(b). In contrast to Fig. 1, the time sequence of Fig. 3(b) images only the specific mode under consideration. A singlenode, non-axially symmetric mode is imaged in Fig. 3(c) (with left, amplitude and right phase) and Fig. 3(d) (snapshots of this mode in the time domain). We observe, for the single-node non-axial mode, a systematic striking behavior, illustrated in Fig. 4 for a $4 \mu \mathrm{m}$,

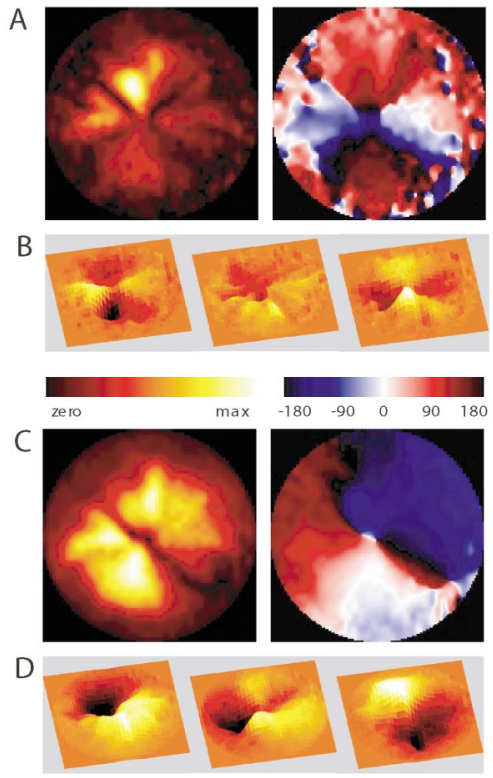

FIG. 3 (color). Nonaxially symmetric modes. (a) on the left the amplitude and on the right the phase of the mode with $1.5 \mathrm{GHz}$ is displayed. (b) Back transforming the images in (a) produces a temporal sequence similar to the one in Fig. 1, but containing only the mode under investigation. Three images of this sequence also available as animated online movie [12], are given in (b). Time interval between middle (right) and left image:150 (360) ps. This mode consists of four regions, oscillating in pairs in phase. (c): On the left the amplitude and on the right the phase of a mode with $1.9 \mathrm{GHz}$. This mode has only one in-plane node dividing a region of roughly equal Fourier amplitude but roughly opposite phase. (d) Back transformation of the images in (c) produces the temporal evolution of this mode, illustrated by three images (time interval between the middle (right) image and the left image: 120 (270) ps and available as an animated movie online [12]. This mode consists of two regions divided by a node and oscillating with opposite phase. respectively, $3 \mu \mathrm{m}$ diameter disk. As in Fig. 3, the Fourier-amplitude is on the left of Figs. 4(a) and 4(c), the Fourier phase on the right, and the reconstructed time sequences in Fig. 4(b) and 4(d). In Fig. 4(a) the node is less pronounced on one side, or almost suppressed 4(c). The phase changes in both cases, but, in particular, in Fig. 4(c), almost continuously along a circular path surrounding the center. Correspondingly, the time domain sequences 4(b) and 4(d) reveal that this mode is traveling along a circular trajectory surrounding the core. We explain this motion by noticing that the symmetry of this problem allows the existence of two mutually orthogonal degenerate single-node modes which can be simultaneously excited, their relative weight depending on the actual geometry of the tipping pulse. Superposition of such modes can produce the observed "circularly polarized" eigenmode, just as, by analogy, linearly polarized light can be superposed to yield circular polarization. In this specific sample, our electron microscopy images reveal that the $3 \mu \mathrm{m}$ disk is slightly displaced from the coil center while the $6 \mu \mathrm{m}$ disk (shown in Fig. 3) is not:

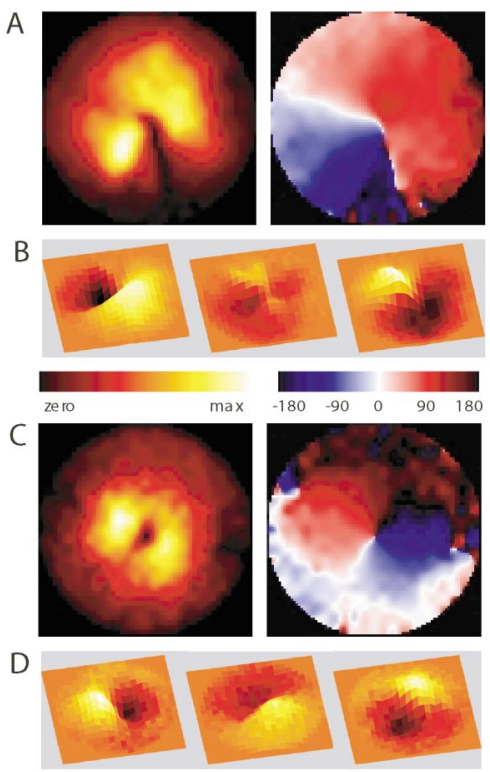

FIG. 4 (color). Circularly polarized mode. (a) The singlenode nonaxially symmetric mode in a $4 \mu \mathrm{m}$ disk, occurring at $2.5 \mathrm{GHz}$. The node is more pronounced on one side, where the $\pi$-phase jump is also clearly visible. (b) The back transformed temporal sequence (see also online animated movie [12]) is given in (b). The time interval between the middle (right) and left images is 80 (160) ps. (c) The single-node nonaxially symmetric mode in a $3 \mu \mathrm{m}$ disk occurs at 2.8 GHz. The node is weak and the phase (right) changes gradually along a trajectory surrounding the core center. Correspondingly, the temporal sequence obtained by back transformation - given in (d) after a time interval of 125 and $250 \mathrm{ps}$ and also available online as an animated movie [12] - consists of a bright-dark doublet rotating around the disk center. 


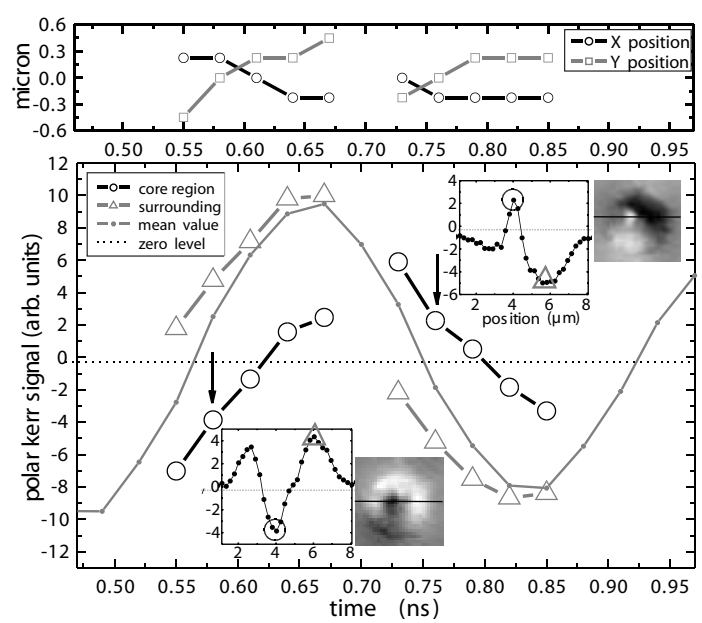

FIG. 5. The core region. Top: plot of the coordinates of the central part with respect to the geometrical image center $(X=$ $0, Y=0)$, in units of $\mu \mathrm{m}$. Bottom: the continuous line indicates the average signal over the disk, and provides us with a check of the zero line (dotted), obtained from the nonmagnetic surroundings. The triangles give the strength of the magnetization surrounding the core, as derived from line scans of the images of the type indicated. The circles give the strength of the magnetization in the core region, which is seen to become negative and positive. The signal also deviates systematically from the zero line expected for axially symmetric dipolar modes. The data from the line scans shown are marked with arrows.

this explains why the "circularly polarized" mode is more pronounced in Fig. 4 than in Fig. 3.

To explain the modes in Fig. 3 and 4, we recall that non-axially symmetric magnetostatic modes in an infinite circular disk are $\propto e^{i n \phi}, n$ being an integer $(n=0$ for axially symmetric modes) and $\phi$ the in-plane polar angle[15]. Our micromagnetic simulations reproduce both frequencies very accurately. A more detailed theory might elucidate this interesting finding.

We now proceed to the motion of the well defined, circular element seen in some of the images of Fig. 1 to reside in the vicinity of the center of the disk. From line scans across the image, this circular element appears as a clear peak (see insets in the bottom part of Fig. 5) with a width of the order of the spatial resolution. The location of its maximum can be determined with an accuracy of about $0.2 \mu \mathrm{m}$ and is seen to move with time (Fig. 5, top). Furthermore, its $z$-magnetization is oscillating with time (Fig. 5, bottom, circles). Most strikingly, at some times, the central part of the element assumes negative $M_{z}$ values, i.e., it is oppositely magnetized with respect to the immediate surroundings (bottom, triangles). Notice that the eigenmodes in this dipolardominated regime are expected to have zero amplitude at the center of the disk[10]. We do not have an immediate explanation for the negative $M_{z}$ magnetization at the center. Notice that our micromagnetic simulations, which include the excitation of non-axially symmetric modes by a non-uniform pulse, appear to reveal this most remarkable process as well.

In summary, we have studied the excitation spectrum of simple magnetic vortex state structures with high accuracy and have identified several eigenmodes of the system. Micromagnetic simulations show excellent agreement with our experimental findings.

Financial support by the Deutsche Forschungsgemeinschaft through the Priority Programme 1133 "Ultra Fast Magnetization Processes", the Forschergruppe FOG370/2-1 and the Swiss National Fund is gratefully acknowledged.

[1] W. K. Hiebert, A. Stankiewicz, and M. R. Freeman, Phys. Rev. Lett. 79, 1134 (1997).

[2] J. P. Park et al., Phys. Rev. B 67, 020403(R) (2003), and references therein.

[3] R. J. Hicken, A. Barman, V. V. Kruglyak, and S. Ladak, J. Phys. D 36, 2183 (2003), and references therein.

[4] T.Shinjo, T. Okuno, R. Hassdorf, K. Shigeto, T. Ono, Science 289, 930 (2000).

[5] J. Raabe, R. Pulwey, R. Sattler, T. Schweinböck, J. Zweck, and D. Weiss, J. Appl. Phys. 88, 4437 (2000).

[6] A. Wachowiak, J. Wiebe, M. Bode, O. Pietzsch, M. Morgenstern, and R. Wiesendanger, Science 298, 577 (2002).

[7] R. Höllinger, A. Killinger, and U. Krey, J. Magn. Magn. Mater. 261, 178 (2003).

[8] Y. Gaididei, T. Kamppeter, F.G. Mertens, and A. R.Bishop, Phys. Rev. B 61, 9449 (2003).

[9] Y. Acremann, C. H. Back, M. Buess, O. Portmann, A. Vaterlaus, D. Pescia, and H. Melchior Science 290, 492 (2000).

[10] M. Buess, Y. Acremann, A. Kashuba, C. H. Back, and D. Pescia, J. Phys. Condens. Matter 15, 1093(R) (2003).

[11] Y. Acremann, A. Kashuba, M. Buess, D. Pescia, and C. H. Back, J. Magn. Magn. Mater. 239, 346 (2002).

[12] See EPSPD Document No. E-PRLTAO-93-024431 for animated online movies showing sequences in the figures. A direct link to this document may be found in the online article's HTML reference section. The document may also be reached via the EPAPS homepage (http:// www.aip.org/pubdervs/epaps.html) or from ftp.aip.org in the directory /epaps/. See the EPAPS homepage for more information.

[13] The LLG code can be found at http://llgmicro.home.mindspring.com.

[14] M. Buess et al. (to be published).

[15] M. Sparks, Solid State Commun. 8, 731 (1970). 